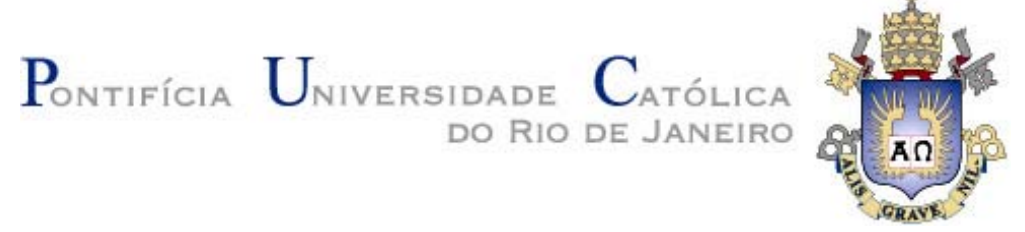

Gustavo Lopes Mourad

\title{
Um Framework para a Construção de Mediadores Oferecendo Eliminação de Duplicatas
}

\section{Dissertação de Mestrado}

Dissertação apresentada ao Programa de Pós-graduação em Informática da PUC-Rio como requisito parcial para obtenção do título de Mestre em Informática.

Orientador: Prof. Karin Breitman

Rio de Janeiro

Setembro de 2010 


\title{
Gustavo Lopes Mourad
}

\section{Um Framework para a Construção de Mediadores Oferecendo Eliminação de Duplicatas}

\begin{abstract}
Dissertação apresentada como requisito parcial para a obtenção do grau de Mestre pelo Programa de Pós-graduação em Informática do Departamento de Informática do Centro Técnico e Científico da PUC-Rio. Aprovada pela Comissão Examinadora abaixo assinada.
\end{abstract}

\author{
Prof. Karin Breitman \\ Orientador \\ Departamento de Informática - PUC-Rio
}

Prof. Marco Antonio Casanova

Departamento de Informática - PUC-Rio

Prof. Simone Diniz Junqueira Barbosa

Departamento de Informática - PUC-Rio

Prof. José Eugenio Leal

Coordenador Setorial do Centro

Técnico Científico - PUC-Rio

Rio de Janeiro, 14 de Setembro de 2010 
Todos os direitos reservados. É proibida a reprodução total ou parcial do trabalho sem autorização da universidade, do autor e do orientador.

\section{Gustavo Lopes Mourad}

Graduou-se em Engenharia de Computação pela Pontifícia Universidade Católica do Rio de Janeiro. Desenvolveu como projeto de mestrado um framework para enriquecimento de bases de dados com recursos da Deep Web.

Ficha Catalográfica

Mourad, Gustavo L.

Um Framework para a Construção de Mediadores Oferecendo Eliminação de Duplicatas / Gustavo Lopes Mourad; orientador: Karin Breitman. - Rio de Janeiro : PUC-Rio, Departamento de Informática, 2010

v., 69 f: il. ; 29,7 cm

1. Dissertação (mestrado) - Pontifícia Universidade Católica do Rio de Janeiro, Departamento de Informática.

Inclui referências bibliográficas.

1. Informática - Dissertação. 2. Detecção de duplicatas. 3. Resolução de entidades . 4. Integração de Dados 5. Deep Web. I. Breitman, Karin. II. Pontifícia Universidade Católica do Rio de Janeiro. Departamento de Informática. III. Título

CDD: 004 
Dedico este trabalho a meus pais e avós pelo apoio aos estudos em todas as fases da minha vida, minha esposa Rachel e minha irmã. 


\section{Agradecimentos}

A minha orientadora, Dr. Karin Breitman, por ter me aceito e acreditado no meu trabalho. Sua competência, dedicação e paciência foram fundamentais para a realização desta dissertação.

Aos professores do Departamento de Informática da PUC-Rio pelas aulas, conselhos e questões estimulantes.

A todos os colegas de curso e trabalho que sempre me apoiaram com coleguismo, respeito e amizade.

Por fim, agradeço à CAPES e PUC-Rio pelo apoio financeiro e ao Departamento de Informática da PUC-Rio pela excelente formação. 


\section{Resumo}

Mourad, Gustavo L.; Breitman, K. Um Framework para Construção de Mediadores Oferecendo Eliminação de Duplicatas. Rio de Janeiro, 2010. 69p. Dissertação de Mestrado - Departamento de Informática, Pontifícia Universidade Católica do Rio de Janeiro.

À medida em que aplicações web que combinam dados de diferentes fontes ganham importância, soluções para a detecção online de dados duplicados tornam-se centrais. A maioria das técnicas existentes são baseadas em algoritmos de aprendizado de máquina, que dependem do uso de bases de treino criadas manualmente. Estas soluções não são adequadas no caso da Deep Web onde, de modo geral, existe pouca informação acerca do tamanho das fontes de dados, da volatilidade dos mesmos e do fato de que a obtenção de um conjunto de dados relevante para o treinamento é uma tarefa difícil. Nesta dissertação propomos uma estratégia para extração (scraping), detecção de duplicatas e incorporação de dados resultantes de consultas realizadas em bancos de dados na Deep Web. Nossa abordagem não requer o uso de conjuntos de testes previamente definidos, mas utiliza uma combinação de um classificador baseado no Vector Space Model, com funções de cálculo de similaridade para prover uma solução viável. Para ilustrar nossa proposta, nós apresentamos um estudo de caso onde o framework é instanciado para uma aplicação do domínio dos vinhos.

\section{Palavras-chave}

Detecção de Duplicatas; Resolução de Entidades; Integração de dados; Deep Web 


\section{Abstract}

Mourad, Gustavo L.; Breitman, K. (advisor). A Framework for the Construction of Mediators Offering Deduplication. Rio de Janeiro, 2010. 69p. MSc Dissertation - Departmento de Informática, Pontifícia Universidade Católica do Rio de Janeiro.

As Web applications that obtain data from different sources (Mashups) grow in importance, timely solutions to the duplicate detection problem become central. Most existing techniques, however, are based on machine learning algorithms, that heavily rely on the use of relevant, manually labeled, training datasets. Such solutions are not adequate when talking about data sources on the Deep Web, as there is often little information regarding the size, volatility and hardly any access to relevant samples to be used for training. In this thesis we propose a strategy to aid in the extraction (scraping), duplicate detection and integration of data that resulted from querying Deep Web resources. Our approach does not require the use of pre-defined training sets, but rather uses a combination of a Vector Space Model classifier with similarity functions, in order to provide a viable solution. To illustrate our approach, we present a case study where the proposed framework was instantiated for an application in the wine industry domain.

\section{Keywords}

Duplicate Detection; Entity Matching; Data Integration; Deep Web 


\section{Sumário}

1 Introdução 12

1.1 Objetivo 13

1.2 Contribuições 14

1.3 Resumo 15

2 Conceitos Básicos 16

2.1 Recuperação de Dados da Web 16

2.2 Construção de rastreadores (crawlers) 18

2.3 Alinhamento de Esquemas - Schema Matching 20

2.4 Resolução de entidades - Entity Matching 20

2.5 Funções de Similaridade entre Cadeias de Caracteres 24

2.6 Classificação 29

2.7 Resumo 32

3 Estratégia para o enriquecimento de informações 34

3.1 Busca de informações na Deep Web 35

3.2 Incorporação de novas informações a partir da
identificação de duplicatas

3.3 Escopo do trabalho 39

$\begin{array}{lll}3.4 & \text { Resumo } & 40\end{array}$

4 Framework Proposto para Construção de Mediadores 41

4.1 Visão Geral do Processo do Framework Proposto 41

4.2 Arquitetura Proposta 44

4.3 Resumo 48

5 Winetag.com.br: um estudo de caso 49

5.1 Metodologia de trabalho 50

5.2 Resultados 58

5.3 Resumo 62

6 Trabalhos relacionados 63

6.1 Rastreadores para Deep Web 63

6.2 Resolução de Entidades 63

6.3 Comparação de resultados 64 
7 Conclusão $\quad 65$

7.1 Contribuições 65

$\begin{array}{lll}7.2 & \text { Trabalhos Futuros } & 66\end{array}$

$8 \quad$ Referências Bibliográficas $\quad 67$ 


\section{Lista de Figuras}

Figura 1 - Vista conceitual da Deep Web [17] 12

Figura 2 - Classificação de Extratores Web [23] 17

Figura 3 - Rastreador (crawler) tradicional [32] 18

Figura 4 - Rastreador (crawler) de Deep Web [32] 19

Figura 5 - Visão geral do algoritmo de similaridade de MARLIN [6] 23

Figura 6 - Representação gráfica de 2 documentos no VSM 27

Figura 7 - Exemplo de execução do KNN 30

Figura 8 - Exemplo de árvore de decisão 31

Figura 9 - Exemplo de estrutura básica de nós de uma rede neural 32

Figura 10 - Visão geral do processo de enriquecimento de informações

Figura 11 - Algoritmo para a busca de informações na Deep Web 36

Figura 12 - Algoritmo para o resolução de entidades 38

Figura 13 - Passos 1, 2 (a) e 3 (b) do processo (estratégia de busca) 42

Figura 14 - Passos 4 e 5 do processo (incorporação) 43

Figura 15 - Diagrama de Classes $\quad 45$

Figura 16 - Diagrama de Componentes do Framework 48

Figura 17 - Exemplo de fonte de dados 53

Figura 18 - Exemplo elementos identificados pelo Developer Tools 53

Figura 19 - Elementos destacados no código-fonte 54

Figura 20 - Diagrama de classes para instância de vinhos 57

Figura 21 - Winetag.com.br: mashup com dados da Deep Web 58 
True genious resides in the capacity for evaluation of uncertain, hazardous, and conflicting information. 